\title{
Antibacterial Properties of Nano-particles in Dental Restorative Materials: Literature Review
}

\author{
Isra Aljubour *, Ala Aljubour \\ General Dentist, Ministry of Health, Riyadh, KSA \\ Corresponding author: Isra Aljubour; dr_esra86@hotmail.com
}

Received 24 September 2020;

Accepted 12 October 2020;

Published 18 October 2020

\begin{abstract}
Nanotechnology has been receiving a great importance because of its ability to modulate materials into their nano size. This has drastically led to many changes in chemical, physical and optical properties of almost all metals. The aim of the present literature review is to examine and quantitatively analyze the current evidence for the addition of different nanoparticles into dental restorative materials, to determine whether their incorporation increases the antibacterial/antimicrobial properties of the materials. The incorporation of nanoparticles into dental restorative materials was a favorable option we found during our review; the antibacterial activity of nanoparticle-modified dental materials was significantly higher compared with the original unmodified materials, $\mathrm{TiO}_{2}$ nanoparticles providing the greatest benefits. However, the high heterogeneity among the articles reviewed points to the need for further research and the application of standardized research protocols.
\end{abstract}

Keywords: dentistry, nano-particles, antibacterial, review, restorative

\section{Introduction}

Restorative dentistry is mainly based on adhesive dentistry in recent years. It has been known that resin-based materials are the best recommended choice for restoration procedures ${ }^{[1]}$. Nevertheless, it has been observed that this type of materials has higher tendency to accumulate more than other types especially dental hard tissues as enamel ${ }^{[2-4]}$.

On of the back draws of dental restorative materials is that they suffer from inherent problems which develops over time. And nearly half of all dental restorations don't continue for more than ten years. It has been estimated that $50-70 \%$ of people tend to replace them ${ }^{[1,4]}$. The main reason why they fail is the colonization by pathogenic organisms that produce caries at the restoration site. Consequently, this causes acid attack and further development of secondary caries. This, in turn, reduces the durability of the restoration ${ }^{[5-6]}$.

This problem is also discussed in the literature of orthodontic applications. The problem ensues because the orthodontic apparatus facilitates the accumulation and proliferation of cariogenic bacteria ${ }^{[1,7]}$. This produces an organic acid which causes enamel demineralization which manifests as white spot lesions (WSL). This is the first sign of demineralization ${ }^{[8-10]}$.

WSL constitutes a major complication in patients undergoing orthodontic treatment with fixed apparatus ${ }^{[11]}$, being its prevalence over $50 \%{ }^{[12]}$. Although non-cavitated lesions are reversible most of the time, they could be potentially irreversible if not diagnosed early or when there is a lack of lesion monitoring
[13]. In those cases, preventative measures that do not require patient compliance may be more effective ${ }^{[11]}$.

Given that bacteria are mainly responsible for shortening restorations' functional life and the appearance of WSL ${ }^{[1]}$, an effective method for preventing enamel demineralization and the appearance of cavitated lesions is the use of dental materials that are resistant to bacterial accumulation and ideally, materials that suppress bacterial activity at the tooth-restoration interface ${ }^{[1,14]}$. Numerous attempts have been made to develop dental restorative materials that offer good long-term behavior and antibacterial activity without sacrificing their mechanical properties ${ }^{[14-15]}$

The most important advance in the field of dental materials has been the introduction of nanotechnology ${ }^{[16]}$, which represents a promising area of research in dentistry, mainly aimed at improving dental materials' antibacterial and mechanical properties ${ }^{[17-18]}$.

Numerous studies have focused on evaluating the antibacterial properties of various nanoparticles and investigating the possibility of incorporating these nanoparticles into dental restorative materials in order to produce restoration materials that prevent bacterial accumulation and so the appearance of secondary caries ${ }^{[12,16,19]}$.

Although a great deal of research has been produced on this subject, it is subject to great variability in terms of the diversity of nanoparticle types assayed and the methodologies applied. To date, no systematic review has been conducted of published research into the properties of nanoparticle-modified dental restorative materials.

Given the increasing interest in nanotechnology in the field of adhesive dentistry, the aim of the present literature review is to 
examine the current evidence for the incorporation of different nanoparticles into dental restorative materials in order to enhance their antibacterial properties.

\section{Nano-structured materials and their applications}

Nanotechnology includes the integration of these nano-scale structures into larger material components and systems, keeping the control and construction of new and improved materials at the nanoscale. In addition, these nano-materials also present different interesting morphologies such as spheres, tubes, rods and prisms. Inorganic nanoparticles including the ones based on metal oxides (zinc oxide, iron oxide, titanium dioxide and cerium oxide), metals (gold, silver and iron, copper, and magnesium), and quantum dots (cadmium sulfide and cadmium selenide) ${ }^{[20-23]}$. Additionally, silicon dioxide and aluminum oxide nanoparticles have been used ${ }^{[23]}$. Alginate nano-materials can also be used as antimicrobial agents ${ }^{[24]}$. Mixtures of different phases are also manufactured. Silver has come up but silver nanoparticles have been proved to be most effective as they have good antimicrobial efficacy against bacteria, viruses, and other eukaryotic microorganisms ${ }^{[20]}$.

As a result of their small size, nanoparticles may offer other advantages to the biomedical field through improved biocompatibility ${ }^{[25]}$. Additionally, it appears that bacteria are far less likely to acquire resistance against metal nanoparticles than other conventional and narrow-spectrum antibiotics. This is thought to occur because metals may act on a broad range of microbial targets, and many mutations should occur for microorganisms to resist their antimicrobial activity. Shape may also affect the activity of nanoparticles ${ }^{[26]}$.

Other metals have been used for centuries as antimicrobial agents. Copper, gold, titanium, and zinc have attracted particular attention, with each having different properties and spectra of antimicrobial activity ${ }^{[27-28]}$. The broad antibacterial activity of nano-silver reduces patient infection, dependence on antibiotic use, and associated costs. There is room for improvement in stabilizing and prolonging the antibacterial effects of nano-silver coatings for medical applications to prevent infection and inflammation. Finally, with the widespread adoption of nano-silver, several concerns about toxicity remain and need to be addressed ${ }^{[29]}$. The use of silver has been severely limited by the toxicity of silver ions to humans. However, nanotechnology has facilitated the production of smaller silver particles with increasing large surface area-tovolume ratios, greater efficacy against bacteria ${ }^{[30]}$ and, most importantly, lower toxicity to humans ${ }^{[31]}$. The mechanisms underlying the impressive biological properties of nano-silver are still not understood and this is a priority for future research in vivo [29].

Both silver and titanium particles were introduced into dental composites, to introduce antimicrobial properties and enhance biocompatibility of the composites ${ }^{[25,32-33]}$. Nano-silver, comprising silver nanoparticles, is attracting interest for a range of biomedical applications owing to its potent antibacterial activity. It has recently been demonstrated that nano-silver has useful antiinflammatory effects. Silver nanoparticles, or nano-silver, are clusters of silver atoms that range in diameter from 1 to $100 \mathrm{~nm}$ and are attracting interest as antibacterial and antimicrobial agents for applications in medicine ${ }^{[29]}$. Moreover, nano-silver exhibits remarkable biological properties, such as antiviral activities ${ }^{[34-35]}$. The action of nano-silver depends on the inhibition of fusion or entry of the virus into the host cell, since blocking HIV entry into its target cells can lead to the suppression of viral infectivity, replication, and cytotoxicity induced by virus-cell interaction ${ }^{[36]}$. In addition to fusion inhibitors, virucidal agents are also urgently needed for HIV/AIDS prevention because they directly inactivate virus particles (virions), thus preventing the completion of the virus replication cycle. Virucidal agents differ from virustatic drugs in that they act directly and rapidly by lysing viral membranes on contact or by binding to virus coat proteins ${ }^{[37]}$. Silver nanoparticles are effective virucides as they inactivate HIV particles in a short period of time, exerting their activity at an early stage of viral replication (entry or fusion) and at post-entry stages ${ }^{[38]}$.

The antimicrobial property of silver is related to the amount of silver and the released rate of silver. Silver in its metallic state is inert, but it reacts with the moisture in the skin and the fluid of the wound and gets ionized. The ionized silver is highly reactive, as it binds to tissue proteins and brings structural changes in the bacterial cell wall and nuclear membrane, leading to cell distortion and death ${ }^{[39]}$. The possible mechanisms underlying the action of metallic silver, silver ions and silver nanoparticles have been proposed according to the morphological and structural changes found in the bacterial cells ${ }^{[34]}$. The effect of silver ions on bacteria can be observed by the structural and morphological changes. It is believed that silver ions interact with the three main components of bacterial cells to produce the bactericidal effect: the peptidoglycan cell wall ${ }^{[40-41]}$ and plasma membrane ${ }^{[42]}$, bacterial DNA ${ }^{[43]}$, and bacterial proteins, especially enzymes involved in vital cellular processes such as the electron transport chain ${ }^{[40]}$. It is reported that silver ions cause the lysis of bacterial cells ${ }^{[33]}$.

\section{Nanoparticles in dental applications}

Most dental treatments become necessary when pathogenic germs colonize the dentine and enamel, the marginal gaps between the dentine and enamel and dental restorations, restoration and prosthetic materials as well as the neighboring soft tissue ${ }^{[44]}$. In particular, the bacteria such as S. mutans and S. lactobacilli produce acids, which cause extensive dental caries and severe damage of hard tissues. Thus, when a root canal is filled with known inert filling materials, germs that remain in the canal will gradually cause an inflammatory process after filling, which makes a renewed treatment necessary or leads to entire loss of teeth. Antimicrobial dental materials are frequently used to preclude these destructive treatments ${ }^{[45]}$. The antimicrobial action is most often achieved by adding active antimicrobial ingredients to the dental material. A restorative material that possesses antibacterial properties and inhibits bacterial growth around the restoration would be desirable. As a means of reducing bacterial and fungal adhesion to dental materials and devices, silver nanoparticles are being investigated for a range of possible applications, for example, incorporation into denture materials and orthodontic adhesives ${ }^{[46]}$.

Dental materials with antimicrobial activity such as filling materials, cements, sealants, materials for temporary restorations, coating materials and adhesives have emerged ${ }^{[47]}$. A problem is that the physical and chemical properties of the dental material, such as its mechanical properties or the hardening behavior must not be affected by the addition of the active ingredients. The release of active ingredients in an effective quantity and over an extended and clinically relevant time span must also be ensured ${ }^{[44]}$. The incorporation of silver nanoparticles into bonding adhesives was successful on both physical and antimicrobial levels ${ }^{[48]}$.

Silver ions have been considered as antibacterial components in dental resin composites ${ }^{[49]}$. The modified tissue conditioner combined with silver nanoparticles displayed 
antimicrobial properties against S. aureus, S. mutans and C. albicans incorporated after 24 -h or 72 -h incubation ${ }^{[50]}$. Light cured flowable composite resin materials can be made to function as an antimicrobial product by the addition of silver hydrosol. The silver hydrosol can be released (at a steady rate over time) from the resin composite matrix to reduce the incidence of tooth decay ${ }^{[45]}$. Silver colloid nanoparticles were added to polymeric adhesive to improve the efficiency of electrical conduction ${ }^{[51]}$. Recently, quaternary ammonium poly (ethylene imine) (QA-PEI) nanoparticles were developed for additional antibacterial activity of restorative composite resins. QA-PEI nanoparticles completely inhibited the growth of S. mutans, and their antibacterial activity lasted at least 3 months ${ }^{[52]}$.

The electrical and flexural properties of silver nanvoparticles-filled epoxy composites were improved ${ }^{[53]}$. The incorporation of silver nitrate and silver nanoparticles (AgNPs) significantly reduced the adhesion of $\mathrm{C}$. albicans to the acrylic resin surface, suggesting that AgNPs-combined denture base materials may be a potential approach to prevent denture stomatitis ${ }^{[54]}$. There are a number of factors that need to be considered in silver nanoparticles-filled epoxy composites such as filler concentration, filler shape and size, and filler composition to modify the properties of metal filled polymer composites ${ }^{[53]}$.

Silver-zinc antimicrobial zeolites were added in low percentages to polymethyl methacrylate ${ }^{[55]}$. It can also be used as a valuable alternative to reduce microbial contamination of tissue conditioners, acrylic resin denture bases, and acrylic base plates of removable orthodontic appliances. Zeolites are aluminum silicate crystalline structures. Addition of $2.5 \%$ of zeolites to the materials resulted in decreased flexural strength and impact strength ${ }^{[56-57]}$. Silver zeolite nanoparticles have been incorporated into mouth rinses and toothpastes ${ }^{[56]}$. Now, powdered zinc citrate or acetate has been incorporated to control the formation of dental plaque. Powdered titanium dioxide is also commonly used as a whitener in toothpastes ${ }^{[51]}$. Additionally, nanoparticles can be used effectively in other materials including hydrogels ${ }^{[58]}$.

A variety of permanent dental cements can be impregnated with silver hydrosol including epoxy resin cements, glass ionomer and resin modified glass ionomer cements (used in permanent cement crowns and bridge work). Any number of commonly used permanent dental cements can also be readily combined with the silver hydrosol solution. By adding the silver hydrosol to these cements, one is able to provide a continuous dynamic antimicrobial bacteriostatic environment capable of reducing bacterial bioburden and thus postoperative inflammation, infection and sensitivity, which are particularly important with vital teeth ${ }^{[44]}$. Novel poly quaternary ammonium salt-containing antibacterial glass-ionomer cement was developed. All the poly quaternary ammonium saltcontaining cements showed a significant antibacterial activity, accompanying with a reduction of initial compressive strength. In addition, it was concluded that the experimental cement is a clinically attractive dental restorative due to its high mechanical strength and antibacterial function ${ }^{[59]}$.

Alginate impression powders can be mixed with water that contains silver hydrosol to create an impression material that has antimicrobial activity. This will reduce microbial cross contamination by bacteria, yeasts, other fungi and viruses to the stone model from the infected impression ${ }^{[58]}$. Antimicrobial root canal sealer/cements with the addition of dilute silver hydrosol are useful in permanent obturation of the root canal following removal of the infected pulp and placement of medicaments.

Scanning electron microscope (SEM) observation of the dispersibility of silver-zirconium phosphate (SZP) nano-inorganic antimicrobial agent in silicone denture soft lining materials indicated that the inorganic nano-granules were well distributed in silicone substrate. Element analysis demonstrated the even distribution of zirconium and silver, which confirmed that there were not obvious nano-agglomerates, and in turn verified the excellent dispersibility of SZP in tested silicone ${ }^{[60]}$. Transmission electron microscopy (TEM) analysis and atomic adsorption spectroscopy revealed that silver nanoparticles are compatible with the acrylic formulation and remain well-dispersed in the final material. Silver nanoparticles have no detrimental effect on the photopolymerization kinetics and the incorporation of nanoparticles was found to reduce the gloss of ultraviolet-cured coatings ${ }^{[61]}$.

Research efforts are currently directed towards eliminating or reducing infection of medical devices. Strategies to prevent biofilm formation include physiochemical modification of the biomaterial surface to create anti-adhesive surfaces, incorporation of antimicrobial agents into medical device polymers, mechanical design alternatives, and release of antibiotics ${ }^{[62]}$. In this context, zinc oxide nanoparticles have undergone in vitro testing in biofilm culture test systems. Zinc oxide nanoparticles blended into a variety of composites were shown to significantly inhibit the growth of S. sobrinus biofilm over a three-day test period ${ }^{[27]}$ Kishen et al. ${ }^{[63]}$ demonstrated a reduction in the number of $\mathrm{E}$. faecalis adhered to the dentine on the surface of the root canal treated with cationic antibacterial nano-particulates such as zinc oxide alone or the combination of zinc oxide and chitosan nanoparticulates. In theory, such surface treatment could prevent bacterial recolonization and biofilm formation in vivo.

Particles of a nano- and micro-size based upon the element silicon for the rapid delivery of antimicrobial and anti-adhesive capabilities to the desired site within the oral cavity have received much attention ${ }^{[64]}$. Some companies have used silica (silicon dioxide, $\mathrm{SiO}_{2}$ ) with a particle size within the definition of nanoparticles in toothpastes for many years, and some are now actively seeking new directions in this area through the use of porous silicon/nano-crystalline silicon technology to carry and deliver antimicrobials such as triclosan ${ }^{[64]}$.

The mechanical properties of $\mathrm{SiO}_{2}$ nanoparticles were improved even at low filler content ${ }^{[65]}$. Nanoparticle filled dental composites may show an enhanced fracture toughness and adhesion to tooth tissue ${ }^{[66]}$. The use of silica nanoparticles to polish the tooth surface may help protect against damage caused by cariogenic bacteria, presumably because the bacteria can be removed more easily. This has been investigated on human teeth in vivo ${ }^{[67]}$. Modified surfaces were shown to reduce the attachment and growth of C. albicans, with the greatest effect observed with 7and 14-nm particles. Such effects could possibly be attributed to surface topography or slow dissolution of the bound silica. Such treatment has the advantages of being non-toxic, simple to apply, and adaptable to three-dimensional surfaces.

Bioactive glasses of the $\mathrm{SiO}_{2}-\mathrm{Na}_{2} \mathrm{O}-\mathrm{CaO}-\mathrm{P}_{2} \mathrm{O}_{5}$ system have been shown to possess antimicrobial activity through the release of ionic alkaline species over time and are under consideration as dentine disinfectants to offer an alternative to calcium hydroxide. Those in the form of amorphous nanoparticles with a size of 20 to $60 \mathrm{~nm}$ may show an advantage over micron-sized material, because the decrease in glass particle size should increase the ionic release into suspension and enhance antimicrobial efficacy. Antimicrobial activity was assessed against E. faecalis, a pathogen often isolated from root canal infections. The killing efficacy of the nano-sized particles was also significantly higher ${ }^{[68]}$. 
Therefore, nanoparticles might improve the mechanical properties such as wear resistance and surface hardness of dental restorative materials ${ }^{[69]}$. The major difference between nano-metric and micrometric particles is that nanoparticles have significantly larger specific surface area, which greatly facilitates the transfer of load from polymer matrix to nanoparticles ${ }^{[70]}$. As a result, nanoparticle-reinforced hybrid system exhibits higher stiffness and better resistance to wear ${ }^{[70]}$.

\section{Conclusion}

Nanoparticles have come up as one of the most effective antibacterial agents due to their large surface area to volume ratios. They can be used as effective growth inhibitors of various microorganisms. Furthermore, nanomaterials can be modified of achieve better efficiency and to facilitate their applications in different fields such as biomaterials and medicine. The long-term antibacterial, physical and clinical effects of nanoparticles on dental and medical biomaterials should be investigated in future studies.

\section{Ethics approval and consent to participate}

This study is a literature review which didn't include any participants and thus, no need for ethical approval

\section{List of abbreviations}

If abbreviations are used in the text they should be defined in the text at first use, and a list of abbreviations should be provided.

\section{Data Availability}

White Spot Lesion (WSL)

Human Immunodeficiency Virus (HIV)

Acquired Immunodeficiency Syndrome (AIDS)

Deoxyribonucleic Acid (DNA)

Quaternary Ammonium Poly (Ethylene Imine) (QA-PEI)

Silver NanoParticles (AgNPs)

Scanning Electron Microscope (SEM)

Silver-Zirconium Phosphate (SZP)

Transmission Electron Microscopy (TEM)

\section{Funding Statement}

None

\section{Competing Interest}

There is no conflict of interest to declare.

\section{Authors' contributions}

Authors in this manuscript have an equal contribution to this work.

\section{Acknowledgments}

No need for this section.

\section{References}

[1] Welch K., Cai Y., Engqvist H., Strømme M. Dental adhesives with bioactive and on demand bactericidal properties. Dent. Mater. 2010;26:491-499.

[2] Aydin Sevinç B., Hanley L. Antibacterial activity of dental composites containing zinc oxide nanoparticles. J. Biomed. Mater. Res. Part B Appl. Biomater. 2010;94:22-31.

[3] Kasraei S., Sami L., Hendi S., Alikhani M.-Y., RezaeiSoufi L., Khamverdi Z. Antibacterial properties of composite resins incorporating silver and zinc oxide nanoparticles on Streptococcus mutans and Lactobacillus. Restor. Dent. Endod. 2014;39:109-114.

[4] Li F., Weir M.D., Chen J., Xu H.H. Comparison of quaternary ammonium-containing with nano-silver containing adhesivein antibacterial properties and cytotoxicity. Dent. Mater. 2013;29:450-461.

[5] El-Wassefy N.A., El-Mahdy R.H., El-Kholany N.R. The impact of silver nanoparticles integration on biofilm formation and mechanical properties of glass ionomer cement. J. Esthet. Restor. Dent. J. 2018;30:146-152.

[6] Das Neves P.B., Agnelli J.A., Kurachi C., de Souza C.W. Addition of silver nanoparticles to composite resin: Effect on physical and bactericidal properties in vitro. Braz. Dent. J. 2014;25:141-145.

[7] Li F., Li Z., Liu G., He H. Long-term antibacterial properties and bond strength of experimental nano silvercontaining orthodontic cements. J. Wuhan Univ. Technol. Mater. Sci. Ed. 2013;28:849-855.

[8] Ahn S.J., Lee S.J., Kook J.K., Lim B.S. Experimental antimicrobial orthodontic adhesives using nanofillers and silver nanoparticles. Dent. Mater. 2009;25:206-213.

[9] Chambers C., Stewart S.B., Su B., Jenkinson H.F., Sandy J.R., Ireland A.J. Silver doped titanium dioxide nanoparticles as antimicrobial additives to dental polymers. Dent. Mater. 2017;33:e115-e123.

[10] Degrazia F.W., Leitune V.C., Garcia I.M., Arthur R.A., Samuel S.M., Collares F.M. Effect of silver nanoparticles on the physicochemical and antimicrobial properties of an orthodontic adhesive. J. Appl. Oral Sci. 2016;24:404410 .

[11] Wang X., Wang B., Wang Y. Antibacterial orthodontic cement to combat biofilm and white spot lesions. Am. J. Orthod. Dentofac. Orthop. 2015;148:974-981.

[12] Sodagar A., Akhavan A., Hashemi E., Arab S., Pourhajibagher M., Sodagar K., Kharrazifard M.-J., Bahador A. Evaluation of the antibacterial activity of a conventional orthodontic composite containing silver/hydroxyapatite nanoparticles. Prog. Orthod. 2016;17:40.

[13] Paula A.B., Fernandes A.R., Coelho A.S., Marto C.M., Ferreira M.M., Caramelo F., do Vale F., Carrilho E. Therapies for White Spot Lesions-A Systematic Review. J. Evid. Based Dent. Pract. 2017;17:23-38.

[14] Hojati S.T., Alaghemand H., Hamze F., Babaki F.A., Rajab-Nia R., Rezvani M.-B., Kaviani M., Atai M. Antibacterial, physical and mechanical properties of flowable resin composites containing zinc oxide nanoparticles. Dent. Mater. 2013;29:495-505.

[15] Ai M., Du Z., Zhu S., Geng H., Zhang X., Cai Q., Yang $\mathrm{X}$. Composite resin reinforced with silver nanoparticlesladen hydroxyapatite nanowires for dental application. Dent. Mater. 2017;33:12-22. 
[16] Sodagar A., Akhoundi M.S.A., Bahador A., Jalali Y.F., Behzadi Z., Elhaminejad F., Mirhashemi A.H. Effect of $\mathrm{TiO} 2$ nanoparticles incorporation on antibacterial properties and shear bond strength of dental composite used in Orthodontics. Dent. Press J. Orthod. 2017;22:6774.

[17] Allaker R.P., Memarzadeh K. Nanoparticles and the control of oral infections. Int. J. Antimicrob. Agents. 2014;43:95-104.

[18] Garcia-Contreras R., Scougall-Vilchis R.J., ContrerasBulnes R., Sakagami H., Morales-Luckie R.A., Nakajima $\mathrm{H}$. Mechanical, antibacterial and bond strength properties of nano-titanium-enriched glass ionomer cement. J. Appl. Oral Sci. 2015;23:321-328.

[19] Garcia P.P.N.S., Cardia M.F.B., Francisconi R.S., Dovigo L.N., Spolidório D.M.P., de Souza Rastelli A.N., Botta A.C. Antibacterial activity of glass ionomer cement modified by zinc oxide nanoparticles. Microsc. Res. Tech. 2017;80:456-461.

[20] Gong P, Li H, He X, Wang K, Hu J, Tan W, et al. Preparation and antibacterial activity of Fe3O4@Ag nanoparticles. Nanotechnol. 2007;18:604-11.

[21] Retchkiman-Schabes PS, Canizal G, Becerra-Herrera R, Zorrilla C, Liu HB, Ascencio JA. Biosynthesis and characterization of $\mathrm{Ti} / \mathrm{Ni}$ bimetallic nanoparticles. Opt Mater. 2006;29:95-9.

[22] Gu H, Ho PL, Tong E, Wang L, Xu B. Presenting vancomycin on nanoparticles to enhance antimicrobial activities. Nano Lett. 2003;3:1261-3.

[23] Ju-Nam Y, Lead JR. Manufactured nanoparticles: an overview of their chemistry, interactions and potential environmental implications. Sci Total Environ. 2008;400:396-414.

[24] Ahmad Z, Pandey R, Sharma S, Khuller GK. Alginate nanoparticles as antituberculosis drug carriers: formulation development, pharmacokinetics and therapeutic potential. Ind $\mathrm{J}$ Chest Dis Allied Sci. 2005;48:171-6.

[25] Kim JS, Kuk E, Yu KN, Kim JH, Park SJ, Lee HJ, et al. Antimicrobial effects of silver nanoparticles. Nanomed Nanotechnol Biol Med. 2007;3:95-101.

[26] Pal S, Tak YK, Song JM. Does the antibacterial activity of silver nanoparticles depend on the shape of the nanoparticle? A study of the gram-negative bacterium Escherichia coli. Appl Environ Microbiol. 2007;27:1712-20.

[27] Allaker RP. The Use of Nanoparticles to Control Oral Biofilm Formation. J Dent Res. 2010;89:1175-1186.

[28] Giertsen E. Effects of mouth rinses with triclosan, zinc ions, copolymer, and sodium lauryl sulphate combined with fluoride on acid formation by dental plaque in vivo. Caries Res. 2004;38:430-5.

[29] Chaloupka K, Malam Y, Seifalian AS. Nanosilver as a new generation of nanoproduct in biomedical applications. Trends Biotechnol. 2010;28:580-8.

[30] Sladkova M, et al. Surface-enhanced Raman scattering from a single molecularly bridged silver nanoparticle aggregate. J Mol Struct. 2009:924-926, 567-70.

[31] Foldbjerg R, et al. PVP-coated silver nanoparticles and silver ions induce reactive oxygen species, apoptosis and necrosis in THP-1 monocytes. Toxicol Lett. 2009;190:156-62.
[32] Jandt KD, Al-Jasser AMO, Al-Ateeq K, Vowles RW, Allen GC. Mechanical properties and radiopacity of experimental glass-silica-metal hybrid composites. Dent Mater. 2002;6:429-35.

[33] Morones JR, Elechiguerra JL, Camacho A, Ramirez JT. The bactericidal effect of silver nanoparticles. Nanotechnol. 2005;16:2346-53.

[34] Rai M, Yadav A, Gade A. Silver nanoparticles as a new generation of antimicrobials. Biotechnol Adv. 2009;27:76-83.

[35] Duran N, Marcarto PD, De Souza GIH, Alves OL, Esposito E. Antibacterial effect of silver nanoparticles produced by fungal process on textile fabrics and their effluent treatment. J Biomed Nanotechnol. 2007;3:2038.

[36] Lara HH, Ayala-Nuñez NV, Ixtepan-Turrent L, Rodriguez-Padilla C. Mode of antiviral action of silver nanoparticles against HIV-1. J Nanobiotechnol. 2010;8:2-10.

[37] Borkow G, Lapidot A. Multi-targeting the entrance door to block HIV-1. Curr Drug Targets Infect Disord. 2005;5:3-15.

[38] Al-Jabri AA, Alenzi FQ. Vaccines, Virucides and Drugs Against HIV/AIDS: Hopes and Optimisms for the Future. Open AIDS J. 2009;3:1-3.

[39] Castellano JJ, Shafii SM, Ko F, Donate G, Wright TE, Mannari RJ, et al. Comparative evaluation of silvercontaining antimicrobial dressings and drugs. Int Wound J. 2007;4:114-22.

[40] Yamanaka M, et al. Bactericidal actions of a silver ion solution on Escherichia coli, studied by energy-filtering transmission electron microscopy and proteomic analysis. Appl Environ Microbiol. 2005;71:7589-93.

[41] Raffi M, Hussain F, Bhatti TM, Akhter JI, Hameed A, Hasan MM. Antibacterial Characterization of Silver Nanoparticles against E. Coli ATCC-15224. J Mater Sci Tech. 2008;24:192-6.

[42] Jung WK, et al. Antibacterial activity and mechanism of action of the silver ion in Staphylococcus aureus and Escherichia coli. Appl Environ Microbiol. 2008;74:2171-8.

[43] Shrivastava S. Characterization of enhanced antibacterial effects of novel silver nanoparticles. Nanotechnol. 2007;18:225103-12.

[44] Subba Rao CVS, Vanajasan PP, Chandana VS. Scope of Biomaterials in Conservative Dentistry and Endodontics. Trends Biomater Artif Organs. 2011;25:75-8.

[45] Li Z, Lee D, Sheng X, Cohen RE, Rubner MF. Two-level antibacterial coating with both release-killing and contact-killing capabilities. Langmuir. 2006;22:9820-3.

[46] Monteiro DR, Gorup LF, Takamiya AS, Ruvollo-Filho AC, de Camargo ER, Barbosa DB. The growing importance of materials that prevent microbial adhesion: antimicrobial effect of medical devices containing silver. Int J Antimicrob Agents. 2009;34:103-10.

[47] Ahn SJ, Lee SJ, Kook JK, Lim BS. Experimental antimicrobial orthodontic adhesives using nanofillers and silver nanoparticles. Dent Mater. 2009;25:206-13.

[48] Moszner N, Salz U. Recent Developments of New Components for Dental Adhesives and Composites. Macromol Mater Eng. 2007;292:245-71. 
[49] Ahn S, Lee S, Kook J, Lim B. Experimental antimicrobial orthodontic adhesives using nanofillers and silver nanoparticles. Dent Mater. 2009;25:206-13.

[50] Herrera M, Carrion P, Baca P, Liebana J, Castillo A. In vitro antibacterial activity of glass-ionomer cements. Microbios. 2001;104:141-8.

[51] Okita N, Orstavik D, Orstavik J, Ostby K. In vivo and in vitro studies on soft denture materials: microbial adhesion and tests for antibacterial activity. Dent Mater. 1991;7:155-60.

[52] Lee HH, Chou KS, Shih ZW. Effect of nano-sized silver particles on the resistivity of polymeric conductive adhesives. Inter J Adhes Adhes. 2005;25:437-41.

[53] Yudovin-Farber I, Beyth N, Nyska A, Weiss EI, Golenser J, Domb AJ. Surface Characterization and Biocompatibility of Restorative Resin Containing Nanoparticles. Biomacromolecules. 2008;9:3044-50.

[54] Mariatti M, Azizan A, See CH, Chong KF. Effect of silane-based coupling agent on the properties of silver nanoparticles filled epoxy composites. Compos Sci Technol. 2007;67:2584-91.

[55] Lee C, Lee M, Nam K. Inhibitory Effect of PMMA Denture Acrylic Impregnated by Silver Nitrate and Silver Nano-particles for Candida Albicans. J Korean Chemic Soci. 2008;52:380-6.

[56] Boldyryeva $\mathrm{H}$, Umeda $\mathrm{N}$, Plaskin OA, Takeda Y, Kishimoto N. Highfluence implantation of negative metal ions into polymers for surface modification and nanoparticle formation. Surf Coat Tech. 2005;196:373-7.

[57] Casemiro LA, Gomes-Martins CH, Pires-de-Souza FdeC, Panzeri H. Antimicrobial and mechanical properties of acrylic resins with incorporated silver-zinc zeolite - Part 1. Gerodontology. 2008;25:187-94.

[58] Kassaee MZ, Akhavan A, Sheikh N, Sodaga A. Antibacterial Effects of a New Dental Acrylic Resin Containing Silver Nanoparticles. J Appli Polym Sci. 2008;110:1699-3.

[59] Lee WF, Tsao KT. Preparation and properties of nanocomposite hydrogels containing silver nanoparticles by ex situ polymerization. J Appl Polym Sci. 2006;100:3653-61.

[60] Xie D, Weng Y, Guo X, Zhao J, Gregory RL, Zheng C. Preparation and evaluation of a novel glass-ionomer cement with antibacterial functions. Dent Mater. 2011;27:487-96.

[61] Sui L, Liu Q, Li C. Dispersibility of SZP Antimicrobial Agents in Silicone Denture Soft Lining Materials and Their Effect on Operational Performance. Biomed Eng (iCBBE) 2010;4:1-4.

[62] Balan L, Schneider R, Lougnot DJ. A new and convenient route to polyacrylate/silver nanocomposites by light-induced cross-linking polymerization. Prog Org Coat. 2008;62:351-7.

[63] Rodrigues LR. Inhibition of bacterial adhesion on medical devices. Adv Exp Med Biol. 2011;715:351-67.

[64] Kishen A, Shi Z, Shrestha A, Neoh KG. An investigation on the antibacterial and antibiofilm efficacy of cationic nanoparticulates for root canal infection. J Endod. 2008;34:1515-20.

[65] Stephen KW. Dentifrices: recent clinical findings and implications for use. Int Dent J. 1993;43(6 S1):549-53.

[66] Du Minhui, Zheng Ying. Modification of silica nanoparticles and their application in UDMA dental polymeric composites. Poly Compos. 2007;28:198-207.

[67] Manhart J, Kunzelman KH, Chen HY, Hickel R. Mechanical properties and wear behaviour of light-cured packable composite resins. Dent Mater. 2000;16:33-40.

[68] Gaikwaad RM, Sokolov I. Silica nanoparticles to polish tooth surfaces for caries prevention. J Dent Res. 2008;87:980-3.

[69] Waltimo T, Brunner TJ, Vollenweider M, Stark WJ, Zehnder M. Antimicrobial effect of nanometric bioactive glass 45S5. J Dent Res. 2007;86:754-7.

[70] Zhao J, Xie D. Effect of Nanoparticles on Wear Resistance and Surface Hardness of Dental Glassionomer Cement. J Compos Mater. 2009;43:2739-51. 